\title{
Formación en investigación y desarrollo de medicamentos: diez años de experiencia (2002-2012)
}

\author{
Fèlix Bosch, Alba Navarro, Laura García-Martín, Josep E. Baños
}

Introducción. Desde el año 2002, la Universitat Pompeu Fabra ha ofrecido un itinerario profesional en industria sanitaria dentro de su licenciatura en biología. El programa combina teoría (clases magistrales, seminarios) con un período de prácticas en instituciones o empresas dedicadas a la investigación farmacéutica. En 2006, se empezó un Máster en Industria Farmacéutica y Biotecnológica inspirado en este curso. El presente artículo describe ambos programas y evalúa las percepciones actuales de los antiguos alumnos sobre la formación recibida, así como su situación profesional actual.

Sujetos y métodos. Se envió un cuestionario por correo electrónico a todos los antiguos alumnos de los que se disponía de su dirección $(n=249)$ preguntando sobre su trabajo actual y la opinión del curso que siguieron. Puntuaron sus opiniones de 0 (muy mal) a 10 (excelente).

Resultados. Contestaron 107 estudiantes (43\%). Las opiniones sobre la formación teórica y las prácticas externas tuvieron una puntuación media de 7,6 y 8,8, respectivamente. La contribución del programa a su trabajo actual obtuvo una media de 8,2. El 90,6\% de los participantes respondieron que volverían a escoger el mismo programa de tener la oportunidad. Entre los ocupados ( $83,8 \%$ de la muestra), el $62,4 \%$ estaba trabajando en empresas del sector privado, el $31,2 \%$ en universidades o institutos de investigación y el 6,5\% realizaba otros trabajos remunerados. Sólo el 5,4\% estaba desempleado y un 10,8\% seguía otros estudios universitarios. El valor medio de satisfacción con su trabajo actual fue de 7,7.

Conclusiones. El programa de formación en I+D farmacéutica fue valorado positivamente. Además, permitió que los estudiantes encontraran un trabajo satisfactorio, un buen indicador de la capacidad de aplicación del programa a las necesidades profesionales del sector.

Palabras clave. Educación médica. Evaluación de programas. Formación de posgrado I+D de medicamentos. Industria farmacéutica. Inserción laboral.

\section{Training in drug research and development: a ten-year experience (2002-2012)}

Introduction. Since 2002, Universitat Pompeu Fabra has been offering a professional track on Health Care Industry in its bachelor in Biology. The syllabus combines theory (lectures, seminars) plus an internship in a company/institution, and it is primarily focused on drug research. In 2006, the University began a Master in Pharmaceutical Industry and Biotechnology inspired on this course. The present article describes both programs and evaluates the current perceptions of former students about these training activities as well as their present jobs.

Subjects and methods. We e-mailed a questionnaire to all former students with known address $(n=249)$ asking about their current job and opinion the course they followed. They rated their opinions from 0 (very bad) to 10 (excellent).

Results. A total of 107 (43\%) students answered the questionnaire. Opinions on the theoretical teaching and internship had mean scores of 7.6 and 8.8, respectively. The contribution of the course to their current job gave a mean score of 8.2 . A total of $90.6 \%$ of respondents would choose the same course again, given the opportunity. Among those working (83.8\% of the sample), $62.4 \%$ were working in private companies, $31.2 \%$ were in universities or research institutes and $6.5 \%$ were working in other paid jobs. Unemployed were $5.4 \%$ of the sample and $10.8 \%$ were following other degrees at universities. Their job satisfaction achieved a mean score of 7.7.

Conclusions. We conclude that our courses in drug research were highly appreciated. Furthermore they also help students to find a satisfactory job, a good indicator of the program's applicability to the needs of workplace.

Key words. Entering the job market. Medical education. Pharmaceutical companies. Postgraduate training in drug R+D. Program evaluation.
Departamento de Ciencias Experimentales y de la Salud; Universitat Pompeu Fabra (F. Bosch, J.E. Baños). Fundación Dr. Antonio Esteve (F. Bosch, A. Navarro, L. García-Martín). Barcelona, España.

Correspondencia:

Dr. Josep E. Baños. Departamento de Ciencias Experimentales y de la Salud. Universitat Pompeu Fabra. Doctor Aiguader, 88. E-08003 Barcelona.

Fax: +34933160901.

E-mail: josepeladi.banos@upf.edu

Nota:

Datos preliminares de este artículo se presentaron en el XXXIV Congreso de la Sociedad Española de Farmacología. San Pedro del Pinatar, Murcia, 16-19 de septiembre de 2013.

Agradecimientos:

A todos los estudiantes participantes en los cursos de formación sobre investigación y desarrollo de medicamentos que se describen en este artículo, en especial a quienes aceptaron responder los cuestionarios de evaluación, y al personal docente, asistencial y de servicios del Departamento de Ciencias Experimentales y de la Salud y de la Facultad de Ciencias de la Salud y de la Vida de la Universitat Pompeu Fabra. También se desea reconocer la colaboración de los docentes y tutores de las diferentes entidades colaboradoras durante las prácticas.

Conflicto de intereses: No declarado.

Competing interests: None declared.

(c) 2015 FEM 


\section{Introducción}

Existe un cierto consenso en considerar el siglo XIX como aquel en que se inicia el descubrimiento de nuevos medicamentos. Ello fue posible gracias a diversos avances científicos, entre los que cabe destacar el desarrollo de la química orgánica, el nacimiento de la farmacología experimental y la disponibilidad de nuevos instrumentos de medida [1]. Esta coincidencia permitió primero el desarrollo de la química extractiva (morfina, quinina, cafeína) y, después, la química sintética con la síntesis de los primeros antitérmicos extraídos del alquitrán de hulla [2].

Aunque en algunos de los descubrimientos decimonónicos no puede olvidarse el hallazgo accidental, la obra de Ehrlich [3] permite la creación de paradigmas dirigidos a una síntesis más o menos racional de nuevos compuestos, como los arsenicales primero y las sulfamidas después, que permitieron la aparición de una industria farmacéutica claramente separada de la química [4]. El descubrimiento de la penicilina y la estreptomicina [5] dio lugar a departamentos de investigación donde se combinaba la búsqueda de nuevas moléculas con la determinación de sus efectos biológicos. Así, en estos departamentos compartían su trabajo los químicos, los bioquímicos, los biólogos y los farmacéuticos, a los que en los años siguientes se unirían los médicos cuando se hizo evidente la necesidad de determinar el efecto de los medicamentos en los humanos a través de los ensayos clínicos [6].

A partir de finales de la década de 1980, la aparición de la tecnología del ADN recombinante y el nacimiento de la ingeniería genética supuso un cambio sustancial en la orientación de la investigación farmacéutica con la aparición de pequeñas empresas biotecnológicas dirigidas a la obtención de sustancias biológicas que pudieran utilizarse en medicina humana y veterinaria [7]. El éxito de Genentech llevó además a la absorción de algunas de ellas por las empresas farmacéuticas tradicionales, lo que desdibujó todavía más la diferencia entre los fármacos de síntesis y los de origen biotecnológico [8]. Así se llegó al siglo XXI, cuando cada vez es más evidente la dificultad de encontrar nuevas moléculas que supongan un cambio sustancial en terapéutica humana.

Este cuadro complejo no hace más que reconocer la necesidad de una formación adecuada en los profesionales que deban dedicarse a la investigación farmacológica. La formación tradicional universitaria en medicina, farmacia, biología, química o biotecnología puede ser insuficiente para comprender y adquirir la competencia necesaria en el mundo complejo de la investigación de medicamentos. Cada una de ellas permite conocer una parte del todo, pero se precisa la adquisición de conocimientos más globales, junto con las competencias básicas para desarrollarse profesionalmente en este ámbito.

La creación del Espacio Europeo de Educación Superior ha permitido la implementación de programas de posgrado con requisitos académicos estrictos y con reconocimiento europeo. En este marco, los másters constituyen una oportunidad para formar aquellos profesionales que precisan las empresas biofarmacéuticas. Los objetivos del presente artículo son describir la experiencia, la opinión y los resultados de inserción profesional de los participantes en un curso de formación de investigación y desarrollo de medicamentos impartido en la Universitat Pompeu Fabra (UPF).

\section{Sujetos y métodos \\ Características del curso de formación en investigación de medicamentos}

En el año 2002 la UPF inició una actividad docente dirigida a la formación específica en investigación y desarrollo de medicamentos. Dicha actividad constituyó parte del quinto curso de la licenciatura en biología dentro del itinerario profesional 'Industria sanitaria' [9]. Su impartición se mantuvo hasta el año 2012 con la implementación del Grado en Biología Humana, que sustituyó a la antigua licenciatura. Desde 2006 hasta la actualidad, esta formación sobre investigación farmacéutica constituye el núcleo principal del Máster en Industria Farmacéutica y Biotecnológica que se imparte en la UPF. El programa dura un curso académico y tiene como objetivo principal formar a los estudiantes en el ámbito de las empresas destinadas a la investigación de medicamentos y productos biotecnológicos que serán utilizados en la prevención y el tratamiento de las enfermedades humanas. La tabla I recoge los objetivos educativos generales del curso, y la tabla II, las competencias que los participantes debían adquirir. La enseñanza combina teoría en diferentes formatos (clases magistrales, seminarios formativos y seminarios de autoaprendizaje) con seis meses de prácticas a dedicación completa en empresas o instituciones del sector. Las tablas III y IV resumen los planes de estudios tanto del itinerario 'Industria sanitaria' dentro de la licenciatura de biología como del Máster en Industria Farmacéutica y Biotecnológica. 
Tabla I. Objetivos educativos generales del Máster en Industria Farmacéutica y Biotecnológica.

Dotar a los estudiantes de los conocimientos generales sobre el proceso de investigación en la industria farmacéutica, así como de las diferentes etapas en que se realiza

Debatir los compromisos éticos que pueden plantearse en un entorno de investigación y desarrollo de productos de elevada importancia social

Presentar las diferentes figuras profesionales a la que los graduados universitarios pueden optar dentro del sector farmacéutico

Facilitar la adquisición de las competencias transversales esenciales en el proceso de comunicación de la industria farmacéutica y biotecnológica

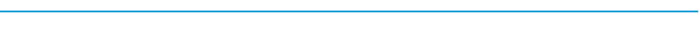

\section{Cuestionario de evaluación del curso}

Durante el mes de junio de 2013 se envió un cuestionario de evaluación del curso por correo electrónico a los antiguos estudiantes (entre 2002 y 2012) de los cuales se disponía de su dirección $(n=249$; 93,3\%). Los encuestados debían valorar su satisfacción sobre diversos aspectos formativos del curso. Además, el cuestionario incluía preguntas sobre su situación profesional actual y su satisfacción al respecto. El grado de satisfacción se determinó mediante una escala numérica con valores entre 0 (muy mala) y 10 (excelente).

\section{Análisis de los datos}

Las respuestas recibidas se introdujeron en una hoja de datos Excel para su análisis. De las variables sobre satisfacción se calcularon las medias, las medianas, las desviaciones estándares y los rangos. Se emplearon diagramas de caja para representar de forma gráfica las puntuaciones. La caja central del diagrama está delimitada por el primer y el tercer cuartil (Q1 y Q3) y representa la distribución del $50 \%$ de los datos. En el interior de la caja se muestra una línea continua que corresponde a la mediana (segundo cuartil, Q2) y una línea discontinua que corresponde a la media. De la caja central se extienden dos segmentos o 'bigotes' verticales que representan los intervalos. Los datos atípicos se muestran como círculos negros.

\section{Resultados}

Entre 2002 y 2012, un total de 309 estudiantes (media de 31 estudiantes por curso) se matricularon al
Tabla II. Competencias que deben adquirir los participantes.

Conocer la estructura de la industria farmacéutica y biotecnológica

Obtener el conocimiento del proceso de investigación y desarrollo (I+D)

Competencias de conocimiento

Conocer el marco legal de los productos derivados de la investigación en empresas del sector biofarmacéutico

Conocer las peculiaridades de los ciclos de producción

Formular hipótesis de trabajo basándose en antecedentes bibliográficos y experimentales

Aprender la dinámica del trabajo en grupo

Competencias metodológicas

Aprender los elementos básicos de la comunicación

Aplicar los conocimientos de búsqueda de la información a una situación real

Aprender a aplicar elementos de decisión a situaciones específicas

Establecer el primer contacto con el mundo de la investigación, la industria privada y el mundo sanitario

Competencias de actitud

Aprender los principios bioéticos del funcionamiento laboral

Conocer el principio de cultura de empresa y de investigación

Tabla III. Plan de estudios del itinerario 'Industria sanitaria' dentro de la licenciatura de biología.

\begin{tabular}{llc}
\hline & & Créditos \\
\hline \multirow{2}{*}{$\begin{array}{l}\text { Asignaturas } \\
\text { obligatorias } \\
\text { del itinerario }\end{array}$} & Fesarrollo de nuevos fármacos y productos sanitarios & 4,5 \\
\cline { 2 - 3 } & Prácticas & 4,5 \\
\hline & Comunicación científica & 4,5 \\
\cline { 2 - 3 } & Neurobiología avanzada y tecnología farmacéutica & 6 \\
\cline { 2 - 3 } $\begin{array}{l}\text { Asignaturas } \\
\text { optativas }\end{array}$ & Epidemiología general & 4,5 \\
\cline { 2 - 3 } & Virología avanzada & 4,5 \\
\cline { 2 - 3 } & Epistemología e historia de la biología y de la medicina & 4,5 \\
\hline
\end{tabular}

programa de formación (itinerario de 'Industria sanitaria' o máster). Como 42 de ellos realizaron el itinerario y posteriormente también el máster, en realidad la docencia se impartió a 267 estudiantes. Durante estos diez años, 69 empresas e instituciones colaboraron para acoger a los estudiantes en prácticas. La mayoría de empresas colaboradoras 
Tabla IV. Plan de estudios del Máster en Industria Farmacéutica y Biotecnológica.

\begin{tabular}{|c|c|c|c|}
\hline & & & $\begin{array}{l}\text { Créditos } \\
\text { ECTS }\end{array}$ \\
\hline \multirow{8}{*}{$\begin{array}{l}\text { Actividades } \\
\text { formativas } \\
\text { obligatorias } \\
\text { de máster }\end{array}$} & \multirow{4}{*}{$\begin{array}{l}\text { Especialidad 1: } \\
\text { industria farmacéutica } \\
\text { y biotecnológica }\end{array}$} & $\begin{array}{l}\text { Desarrollo de nuevos fármacos } \\
\text { y productos sanitarios }\end{array}$ & 5 \\
\hline & & Farmacognosia y tecnología farmacéutica & 5 \\
\hline & & $\begin{array}{l}\text { Introducción a la elaboración de proyecto } \\
\text { de trabajo en la industria farmacéutica }\end{array}$ & 10 \\
\hline & & Trabajo de fin de máster / Prácticas & 30 \\
\hline & \multirow{4}{*}{$\begin{array}{l}\text { Especialidad 2: } \\
\text { biotecnología médica }\end{array}$} & Sistemas biológicos e ingeniería genética & 5 \\
\hline & & $\begin{array}{l}\text { Procesos biotecnológicos aplicables en } \\
\text { la actualidad en los campos de la salud }\end{array}$ & 5 \\
\hline & & $\begin{array}{l}\text { Introducción a la elaboración de proyectos } \\
\text { de trabajo en la industria biotecnológica }\end{array}$ & 10 \\
\hline & & Trabajo de fin de máster / Prácticas & 30 \\
\hline \multirow{9}{*}{$\begin{array}{l}\text { Asignaturas } \\
\text { optativas (deben } \\
\text { escogerse un total } \\
\text { de } 10 \text { créditos ECTS) }\end{array}$} & \multicolumn{2}{|l|}{ Genes y función celular } & 5 \\
\hline & \multicolumn{2}{|l|}{ Comunicación celular } & 5 \\
\hline & \multicolumn{2}{|l|}{ Genomas y sistemas } & 5 \\
\hline & \multicolumn{2}{|c|}{ Organismos modelo en biomedicina } & 5 \\
\hline & \multicolumn{2}{|c|}{ Elementos de biocomputación } & 5 \\
\hline & \multicolumn{2}{|c|}{ Progresos en neurociencias } & 5 \\
\hline & \multicolumn{2}{|l|}{ Comunicación científica } & 5 \\
\hline & \multicolumn{2}{|c|}{ Introducción a la biomedicina } & 5 \\
\hline & \multicolumn{2}{|l|}{ Ciencia en acción } & 5 \\
\hline
\end{tabular}

eran farmacéuticas $(33,3 \%)$, aunque también participaron en el programa centros públicos de investigación, empresas biotecnológicas y organizaciones de investigación bajo contrato - contract research organizations (CRO)-, entre otras (Fig. 1).

En la tabla V se describe el perfil de los 107 estudiantes (43\%) que contestaron el cuestionario. Principalmente se trataba de mujeres que habían cursado la licenciatura de biología en la UPF. Algunos alumnos $(n=24)$ cursaron el máster tras haber realizado el itinerario de 'Industria sanitaria'.

En la figura 2 se muestra la satisfacción de los egresados por las diferentes secciones evaluadas del curso. La satisfacción global del programa de for-
Tabla V. Características generales de la muestra que respondió a la encuesta.

\begin{tabular}{|c|c|c|c|}
\hline & & $n$ & $\%$ \\
\hline \multicolumn{2}{|c|}{ Total de respuestas } & 107 & $43,0^{\mathrm{a}}$ \\
\hline \multirow{2}{*}{ Sexo } & Mujeres & 81 & 75,7 \\
\hline & Hombres & 26 & 24,3 \\
\hline \multirow{2}{*}{ Formación } & Biología UPF & 84 & 78,5 \\
\hline & Otros $^{b}$ & 23 & 21,5 \\
\hline \multirow{3}{*}{$\begin{array}{l}\text { Modalidad } \\
\text { cursada }\end{array}$} & Itinerario 'Industria sanitaria' & 60 & 56,1 \\
\hline & $\begin{array}{l}\text { Itinerario 'Industria sanitaria' } \\
\text { + máster }\end{array}$ & 24 & 22,4 \\
\hline & Máster & 23 & 21,5 \\
\hline
\end{tabular}

a Sobre los 249 estudiantes con los que se contactó (309 matriculados, 267 reales); bioquímica, biotecnología y farmacia.

Tabla VI. Situación laboral de los estudiantes encuestados a .

\begin{tabular}{lcc}
\hline & $n$ & $\%$ \\
\hline Trabajo remunerado & 93 & 83,8 \\
En empresas del sector & 58 & $62,4^{\mathrm{b}}$ \\
En universidades e institutos de investigación & 29 & $31,2^{\mathrm{b}}$ \\
En otras empresas & 6 & $6,5^{\mathrm{b}}$ \\
\hline Cursando estudios universitarios & 12 & 10,8 \\
\hline Buscando trabajo & 6 & 5,4 \\
\hline
\end{tabular}

a Los datos se obtuvieron a partir de las 111 respuestas de los 107 egresados que respondieron el cuestionario enviado por correo electrónico el mes de junio de 2013 (se produjeron cuatro casos con duplicidad de opciones); ${ }^{b}$ Porcentajes calculados dentro del subgrupo que manifestaron disponer de trabajo remunerado.

mación alcanzó una media de $8 \pm 1,4$ (rango: $2-10$ ). El apartado mejor valorado fue el período de prácticas en instituciones del sector (media: $8,8 \pm 1,4$; rango: $2-10)$.

En el momento en que se realizó la encuesta, el $83,8 \%$ de los encuestados disponía de un trabajo remunerado y sólo un 5,4\% estaba en situación de desempleo (Tabla VI). De los primeros, la mayoría $(62,4 \%)$ trabajaba en el sector privado. Estos datos 
están acorde con la puntuación que recibió la contribución del programa de formación a la inserción laboral, una media de 8,2 $\pm 1,5$ (rango: $4-10 ; n=106$ ). Además en general, los egresados mostraron estar satisfechos con su situación laboral actual (media: $7,7 \pm 1,7$; rango: $1-10 ; n=106$ ).

En el último apartado del cuestionario se incluían dos preguntas para recoger más información sobre la satisfacción general con el programa de formación en investigación farmacéutica: ¿elegiría de nuevo este programa de formación? y ¿recomendaría este programa a otras personas? A la primera cuestión, el 90,6\% de los interrogados respondió afirmativamente frente a un 7,5\% que contestó que no $(1,9 \%$ no respondieron esta pregunta). Por otro lado, el 93,5\% recomendaría el curso de formación frente al $3,7 \%$ que no lo recomendaría $(2,8 \%$ no contestó). Seis individuos de los ocho que contestaron que no elegirían de nuevo el programa sí lo recomendarían. Concretamente, cuatro de ellos comentaron que no lo volverían a elegir porque se acabaron dedicando profesionalmente a otro ámbito y, por tanto, les sería más útil otro tipo de formación.

\section{Discusión}

El presente artículo presenta un programa de formación en investigación y desarrollo (I+D) de medicamentos que se ha ofrecido durante diez años para formar profesionales en el ámbito de la industria farmacéutica y biotecnológica. Presenta además los resultados que se han obtenido en este período mediante la determinación del grado de satisfacción de los participantes y de su inserción profesional actual. A continuación se comentan ambos aspectos de manera más detallada.

No existen muchos programas en España de formación específica en $\mathrm{I}+\mathrm{D}$ de medicamentos. Entre las razones que justifican esta baja disponibilidad se encuentran la necesidad de disponer de la cercanía de un tejido empresarial de este sector que permita que los estudiantes realicen una formación práctica adecuada. En España, esta disponibilidad se limita a Barcelona y Madrid, con la excepción singular de Pamplona por la existencia de una universidad con larga tradición en investigación farmacéutica y de un centro de investigación aplicada de medicamentos. Incluso en el ámbito europeo es aún más extraordinario que tales programas ofrezcan datos de sus resultados de formación, especialmente en lo referente a inserción profesional. En países con larga tradición de industrias farmacéuticas, como Gran Bretaña, sí se han publicado algunas experiencias.
Figura 1. Tipo de instituciones vinculadas con la I+D de medicamentos que han participado en el programa práctico. Los porcentajes se han calculado sobre las 69 empresas e instituciones participantes.

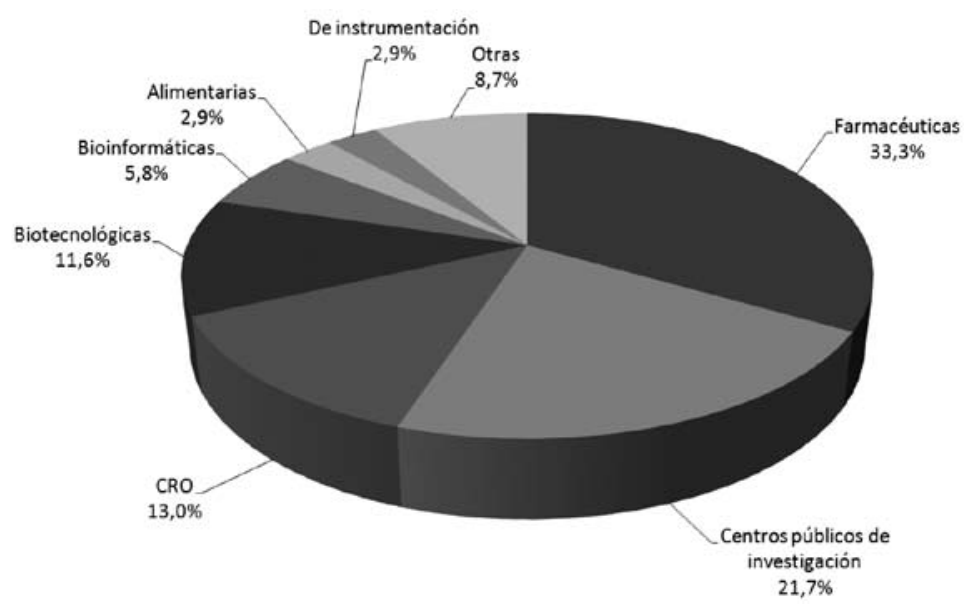

En general, se dirigen a profesionales específicos, como farmacéuticos o farmacólogos [10] o médicos [11], pero tampoco ofrecen resultados de ocupación laboral tras finalizar los programas de formación. Por ello, los datos presentados en el presente artículo tienen el interés adicional de mostrar no sólo la estructura del programa docente, sino también qué ha supuesto para los estudiantes participantes en términos de progresión profesional. Respecto a otros programas analizados, el que presentamos cuenta con un programa de formación en las propias compañías y la posibilidad de una formación opcional en el ámbito propio de la industria farmacéutica o de la biotecnológica, algo especialmente relevante en un momento en que esta última está proporcionando nuevas moléculas y avanza con paso firme en el diseño de nuevos e innovadores fármacos. Creemos, no obstante, que en pocos años no podrán establecerse diferencias entre ambas y deberá hablarse más de empresas biofarmacéuticas, ya que una gran parte de las competencias necesarias de los profesionales serán comunes a ambos ámbitos.

Con respecto al período de formación práctica, sobre el que los estudiantes manifiestan un grado elevado de satisfacción, creemos que es de gran importancia para asegurar un adecuado conocimiento y dominio de lo que el mundo profesional requiere. Para programas de formación avanzada, el empleo de métodos híbridos que combinen una formación teórica con la experiencia del mundo laboral es esencial para todos ellos y, especialmente para el 
Figura 2. Satisfacción con el programa de formación en investigación farmacéutica expresada por los estudiantes que respondieron el cuestionario $(n=107)$. En abscisas se representan los aspectos evaluados mediante la encuesta. A: programa general de formación; B: clases magistrales; C: seminarios autoformativos; D: sesiones de aprendizaje basado en problemas; E: prácticas en instituciones del sector. La satisfacción (en ordenadas) se evaluó mediante una escala numérica con valores entre 0 (muy mala) y 10 (excelente).

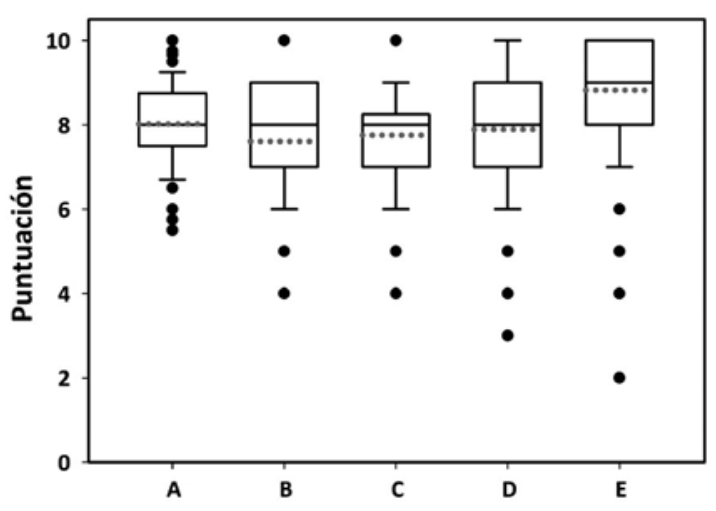

ámbito de $\mathrm{I}+\mathrm{D}$ de medicamentos. Ello se debe a la necesidad de conocer un sector que difícilmente se trata de forma detallada en los planes de estudio actuales por su complejidad y las múltiples funciones y profesionales que en él se desempeñan. No es ya un perfil profesional de farmacéuticos, químicos o médicos, sino que incluye a biólogos, biotecnólogos, ingenieros biomédicos y otros graduados universitarios que pueden desempeñar un papel importante, una vez formados de forma holística en las necesidades de las empresas.

Como ya se ha comentado, un aspecto relevante se refiere a la inserción profesional de los estudiantes en empresas del sector. Consideramos que es un indicador de que el programa cumple con las necesidades de las compañías del ámbito biofarmacéutico, lo que atribuimos tanto a la formación teórica como a la estancia práctica en las empresas. Además, el reconocimiento de los estudiantes a cómo el programa los ha ayudado a encontrar su actual lugar de trabajo y el elevado número de ellos que recomendarían el programa suponen una apreciación positiva de éste, especialmente cuando se realiza años después de haberlo cursado.

Como limitación potencial para la interpretación de los resultados de este estudio debe recordarse que el cuestionario empleado no se encuentra validado y, por ello, podría haber influido en una valoración en exceso positiva de las opiniones de los estudiantes en las preguntas de satisfacción. Sin embargo, sí tenía la validez de contenido suficiente para determinarla de forma adecuada, aunque no podemos descartar tal posibilidad de manera empírica.

En resumen, creemos que los programas de formación en $\mathrm{I}+\mathrm{D}$ de medicamentos son útiles para mejorar los conocimientos en esta área y para favorecer la integración en el mundo laboral de un sector en continuo cambio. La colaboración con las empresas será esencial para garantizar que se mantiene su eficacia en los turbulentos tiempos actuales $\mathrm{y}$, probablemente, futuros.

\section{Bibliografía}

1. Baños JE, Farré M. Historia de la terapéutica farmacológica. In: Principios de farmacología clínica. Barcelona: Masson; 2002; p. 1-13.

2. Sneader W. Drug discovery. A history. Chichester, UK: Wiley; 2005.

3. Bosch F, Rosich L. The contributions of Paul Ehrlich to pharmacology: a tribute on the occasion of the centenary of his Nobel Prize. Pharmacology 2008; 82: 171-9.

4. Chast F. Histoire contemporaine des médicaments. Paris: La Découverte ; 1995.

5. Friedman M, Friedland GW. Alexander Fleming and antibiotics. In: Medicines 10 greatest discoveries. New Haven: Yale University Press; 1998. p. 168-91.

6. Dutta AS. Discovery of new medicines. In Griffin JP, O'Grady J, eds. The textbook of pharmaceutical medicine. 5 ed. Malden: Blackwell Publishers; 2006. p. 4-86.

7. Walsh G. Pharmaceutical biotechnology: concepts and applications. Chichester, UK: John Wiley; 2007.

8. Ho JYR, Gibaldi M. Biotechnology and biopharmaceuticals. Transforming proteins and genes into drugs. Hoboken, NJ: Wiley-Liss; 2003.

9. Aramburu J, Bosch F, Sentí M, Baños JE. Los itinerarios profesionales en biología: un ejemplo de formación académica orientada a la inserción profesional. Educ Med 2006; 9: 23-30

10. Smith JA. An introduction to clinical research and drug development for pharmacy and pharmacology graduate students. J Clin Pharmacol 2002; 42: 867-9.

11. Stanley AG, Jackson D, Barnett DB. The teaching of drug development to medical students: collaboration between the pharmaceutical industry and medical school. Br J Clin Pharmacol 2005; 59: 464-74. 